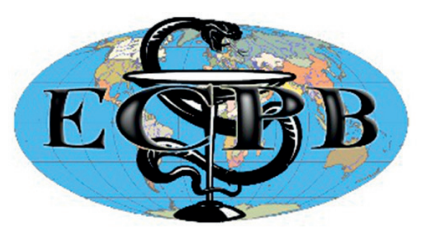

“ЕКСПЕРИМЕНТАЛЬНА ТА КЛІНІЧНА ФІЗІОЛОГІЯ І БІОХІМІЯ” "EXPERIMENTAL AND CLINICAL PHYSIOLOGY AND BIOCHEMISTRY" Науково-практичний журнал/Scientific-practical journal

Наукові статті / Research Articles

\title{
The variety of immune responses to adoptogenetic balneotherapy is strictly deterministic
}

\author{
O.I. MELNYK ${ }^{1}$, Z.D. STRUK ${ }^{2}$ \\ ${ }^{1}$ Danylo Halytskyi National Medical University, L'viv,Ukraine \\ E-mail: omelnyk7@gmail.com
}

${ }^{2}$ Ukrainian Scientific Research Institute of Medicine for Transport, Odessa, Ukraine

Introduction. Four variants of the immune responses to adaptogenic balneotherapy had been identified earlier. In 40,9\% of patients ( $N / N$ cluster), initially the normal immune status (evaluated by 4 parameters of humoral immunity, 5 parameters of cellular immunity as well as 2 parameters of phagocytosis) did not change significantly (Fig. 1). In 31,8\% of patients (N-/N cluster), the lower boundary level of immunity (due to the inhibition of Bactericidity of Neutrophils against Staph. aureus and E. coli) was completely normalized. In $22,7 \%$ of patients ( $\mathrm{S} / \mathrm{S}$ cluster) the moderate suppression of Phagocytosis was reduced but not up to normal. However, in 4,5\% of people (N/S cluster), initially a very increased level of Phagocytosis was transformed into a very decreased level in combination with a slight suppression of cellular immunity and a slight activation of humoral immunity [1,2]. Therefore, the immunotropic effect of balneotherapy on certain individuals is not effective enough and in some cases even unfavorable.

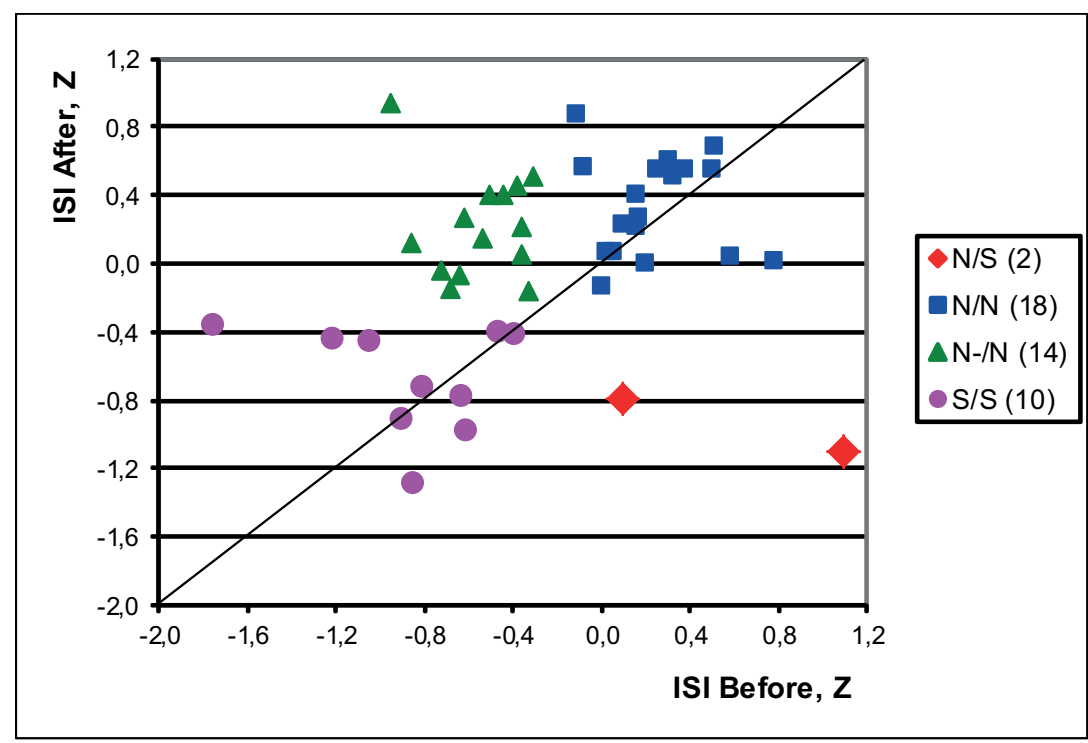

Fig.1. Individual immune status indexes (ISI) before (axis X) and after (axis Y) balneotherapy in members of different clusters of immune responses

(C) Melnyk O.I., Struk Z.D., 2020 
All four variants of immune responses are virtually unmistakably predicted (with an accuracy of $97,7 \%$ ) by a set of 20 predictors including 12 immune blood parameters and one saliva parameter, 4 information parameters, 2 fecal microbiota parameters as well as erythrocyturia [3]. Each type of the immune response is characterized by a specific vegetative, endocrine and metabolic support [4] along with the lines of neuroendocrine-immune modulation [5-9] and functional-metabolic continuum $[10,11]$.

In the next study the discriminant analysis revealed that the constellation of $8 \mathrm{HRV}$ (heart rate variability) and 5 Endocrine parameters as well as gender of a patient predicted the nature of the immune response with an accuracy of $90,9 \%[12]$.

We found out 16 metabolic parameters as the predictors, 3 of them reflected the level of plasma electrolytes, 5 - the level of urine electrolytes, 2 - the levels of plasma and urine urea. Other predictors were the following: very low density lipoprotein cholesterol plasma level, body mass index, fasting gallbladder volume and 30 minutes after cholekinetics, electrokinetic index and a patient's age. These predictors, taken together, determined the nature of the immune response with an accuracy of $95,5 \%$ [13].

Standing on the positions of determinism, we assumed that taking into account all the recorded initial neuro-endocrine and metabolic parameters of the body would allow predicting the immune response of each patient unmistakably. Testing of this hypothesis was the purpose of this study.

Materials and methods. The objects of the observation were 34 men and 10 women aged 24-70, who came to the Truskavets' spa for the treatment of chronic pyelonephritis combined with cholecystitis in the state of remission.

The state of the autonomic nervous system was estimated by parameters of heart rate variability (HRV). We recorded electrocardiogram in II lead (software and hardware complex "CardioLab+HRV" produced by "KhAI-MEDICA", Kharkiv). For the further analysis the following parameters of HRV were selected [14-16]. Temporal parameters (Time Domain Methods): the standard deviation of all $\mathrm{NN}$ intervals (SDNN), the square root of the mean of the sum of the squares of differences between adjacent NN intervals (RMSSD), the percent of interval differences of successive NN intervals greater than $50 \mathrm{~ms}\left(\mathrm{pNN}_{50}\right)$; heart rate (HR), the Mode (Mo), the Amplitude of Mode (AMo), variational sweep (MxDMn) as well as Triangulary Index (TINN). Spectral parameters (Frequency Domain Methods): spectral power (SP), bands of HRV: high-frequency (HF, range $0,4 \div 0,15 \mathrm{~Hz}$ ), low-frequency ( $\mathrm{LF}$, range $0,15 \div 0,04 \mathrm{~Hz}$ ), very low-frequency (VLF, range $0,04 \div 0,015 \mathrm{~Hz}$ ) and ultra low-frequency (ULF, range $0,015 \div 0,003$ $\mathrm{Hz}$ ). On the basis of these parameters the proportion of SP bands (\% of Total Power) and classical indexes: $\mathrm{LF} / \mathrm{HF}, \mathrm{LFnu}=100 \% \cdot \mathrm{LF} /(\mathrm{LF}+\mathrm{HF})$, Centralization Index $=(\mathrm{VLF}+\mathrm{LF}) / \mathrm{HF} ;$ Baevsky Stress Index $(\mathrm{BSI}=\mathrm{AMo} / 2 \cdot \mathrm{Mo} \cdot \mathrm{MxDMn})$ and Baevsky Activity Regulatory Systems Index (BARSI) [14] were calculated as well as the Entropy (h) of HRV [17].

To assess the endocrine status we determined plasma levels of principal adaptation Hormones in the morning on an empty stomach: Cortisol, Testosterone and Triiodothyronine (by ELISA with the use of the analyzer "RT-2100C" and the corresponding sets of reagents from 'Alkor Bio', Xema Co. Ltd and DRG International Inc.).

Then we estimated plasma Lipoprotein spectrum: total cholesterol (by using the direct method after the classic reaction by Zlatkis-Zak) and its content in the composition of a-lipoproteins (the enzyme method by Hiller G. [18] after the precipitation of nota-lipoproteins); pre-B-lipoproteins (by the level of triacylglycerides, by a certain meta-periodate method); $B$-lipoproteins (by a difference between a total cholesterol and cholesterol in composition of $a$-and pre-B-lipoproteins).

We determined also the plasma and daily urine levels of the Electrolytes: calcium (by reaction with arsenase III), magnesium (by reaction with colgamite), 
phosphates (phosphate-molybdate method), chloride (mercury-rhodanidine method), sodium and potassium (flame photometry); Nitrous metabolites: creatinine (Jaffe's color reaction by Popper's method), urea (urease method by reaction with phenolhypochlorite), uric acid (uricase method).

The analyses were carried out according to the instructions described in the manual [19]. The analyzers "Pointe-180" ("Scientific», USA) and "Reflotron" (Boehringer Mannheim, BRD) were used with appropriate sets and a flame spectrophotometer "CF-47".

According to the parameters of electrolyte exchange, the hormonal activity was evaluated: parathyroid by coefficients $(\mathrm{Cap} / \mathrm{Pp})^{0,5}$ and $(\mathrm{Pu} / \mathrm{Cau})^{0,5}$, calcitonin by coefficients $(\mathrm{Cap} \cdot \mathrm{Pp})^{-0,5}$ and $(\mathrm{Cau} \cdot \mathrm{Pu})^{0,5}$ as well as mineralocorticoid by coefficients $(\mathrm{Nap} / \mathrm{Kp})^{0,5}$ and $(\mathrm{Ku} / \mathrm{Nau})^{0,5}$, based on their classical effects and recommendations by I.L. Popovych [9].

The tone and motility of gall-bladder can be estimated by its volume on an empty stomach in the morning and after 5, 15 and 30 min after the ingestion of cholekinetic (50 $\mathrm{ml}$ of $40 \%$ solution of xylitol). The method echoscopy (echocamera "Radmir") was applied.

Norms were borrowed from the database of the Truskavets Scientific School of Balneology.

Results were processed by means of the method of discriminant analysis [20], using the software package «Statistica 5.5».

Results and discussion. Following the accepted algorithm, the forward stepwise method revealed 31 parameters as the predictors (Tables 1 and 2).

Table 1 .

Summary of discriminant function analysis for vegetative,

endocrine and metabolic variables predicting various immune responses Step 31, N of vars in model: 31; Grouping: 4 grps); Wilks' Lambda:

0,000001 ; approx. $\mathrm{F}_{(108)}=13 ; \mathrm{p}<10^{-6}$

\begin{tabular}{|c|c|c|c|c|c|}
\hline Variables currently in the model & $\begin{array}{c}\text { Wilks' } \\
\Lambda\end{array}$ & $\begin{array}{c}\text { Partial } \\
\Lambda\end{array}$ & $\begin{array}{l}\text { F-remo- } \\
\text { ve }(3,5)\end{array}$ & p-level & $\begin{array}{l}\text { Tole- } \\
\text { rancy }\end{array}$ \\
\hline$(\mathrm{Cap} \cdot \mathrm{Pp})^{-0,5}$ as Calcitonin Activity & 00005 & 027 & 60 & ,00024 &, 000 \\
\hline$(\mathrm{Ku} / \mathrm{Nau})^{0.5}$ as Mineralocorticoid activity & ,00007 &, 022 & 75 & 00014 & 003 \\
\hline Magnesium Plasma, mM/L & ,00000 & ,315 & 4 & ,09972 & 069 \\
\hline Baevsky Stress Index normalized by age, Z & ,00006 & ,022 & 73 & ,00015 &, 000 \\
\hline VLD LP Cholesterol, mM/L & ,00026 & ,006 & 300 & ,00000 & 001 \\
\hline Triangulary Index HRV, units & ,00002 & 060 & 26 & ,00176 & 001 \\
\hline Body Mass Index, kg/m² & ,00002 & ,089 & 17 & ,00471 & 021 \\
\hline AMo/MxDMn, units & ,00006 & ,022 & 74 & ,00014 & 000 \\
\hline Gallbladder Volume basal, $\mathrm{mL}$ &, 00006 &, 024 & 69 & ,00017 &, 005 \\
\hline Calcium Excretion, mM/24h & ,00021 & ,007 & 244 & ,00001 & 001 \\
\hline Chloride Urine, $\mathrm{mM} / \mathrm{L}$ & ,00007 & ,021 & 77 & ,00013 & ,003 \\
\hline Cortisol Plasma, nM/L & ,00002 & ,062 & 25 & ,00192 & ,002 \\
\hline Creatinine Excretion, $\mathrm{mM} / 24 \mathrm{~h}$ & 00001 & 225 & 6 & 04472 & ,031 \\
\hline (VLF+LF)/HF HRV &, 00003 & ,049 & 32 & ,00106 & 009 \\
\hline RMSSD HRV, msec &, 00000 & ,296 & 4 & ,08633 & 001 \\
\hline Urea Excretion, $\mathrm{mM} / 24 \mathrm{~h}$ &, 00003 &, 053 & 30 &, 00130 & 001 \\
\hline Sodium Urine, mM/L & ,00001 & , 150 & 9 & 01670 & 018 \\
\hline VLF HRV, \% & ,00006 & ,024 & 69 & ,00017 & 001 \\
\hline LF HRV, msec $^{2}$ & ,00003 & ,051 & 31 & ,00120 & 001 \\
\hline Baevsky Stress Index, ln units & ,00001 & , 135 & 11 & ,01303 & 001 \\
\hline Age, years & ,00001 & , 180 & 8 & ,02611 & 008 \\
\hline SDNN HRV, msec &, 00003 &, 045 & 35 & ,00088 &, 000 \\
\hline Gallbladder Volume after $30 \mathrm{~min}, \mathrm{~mL}$ & ,00001 & ,134 & 11 &, 01270 & 019 \\
\hline $\mathrm{pNN}_{50} \mathrm{HRV}, \%$ & ,00001 & ,218 & 6 &, 04157 & 002 \\
\hline
\end{tabular}




\begin{tabular}{|l|c|c|c|c|c|}
\hline \multicolumn{1}{|c|}{ Variables currently in the model } & $\begin{array}{c}\text { Wilks' } \\
\Lambda\end{array}$ & $\begin{array}{c}\text { Partial } \\
\Lambda\end{array}$ & $\begin{array}{c}\text { F-remo- } \\
\text { ve (3,5) }\end{array}$ & p-level & $\begin{array}{c}\text { Tole- } \\
\text { rancy }\end{array}$ \\
\hline Testosterone normalized by sex\&age, Z &, 00001 &, 226 & 6 &, 04534 &, 012 \\
\hline Calcium Plasma, mM/L &, 00004 &, 036 & 45 &, 00048 &, 002 \\
\hline Urea Plasma, mM/L &, 00002 &, 081 & 19 &, 00372 &, 016 \\
\hline Baevsky Activity Regulatory Systems Index &, 00003 &, 050 & 31 &, 00114 &, 002 \\
\hline Total Power HRV, msec ${ }^{2}$ &, 00001 &, 102 & 15 &, 00656 &, 000 \\
\hline Phosphate Plasma, mM/L &, 00001 &, 126 & 12 &, 01089 &, 003 \\
\hline LFnu HRV, \% &, 00001 &, 273 & 4 &, 07143 &, 025 \\
\hline
\end{tabular}

Table 2 .

Summary of stepwise analysis for vegetative, endocrine and metabolic variables predicting various immune responses. The variables are ranked by Lambda criterion

\begin{tabular}{|c|c|c|c|c|c|}
\hline Variables currently in the model & $\begin{array}{c}\mathrm{F} \text { to } \\
\text { enter }\end{array}$ & p-level & $\Lambda$ & $\begin{array}{c}\text { F- } \\
\text { value }\end{array}$ & p-level \\
\hline$(\mathrm{Cap} \cdot \mathrm{Pp})^{-0,5}$ as Calcitonin Activity & 5,6 & ,003 & ,705 & 5,6 &, 002715 \\
\hline$(\mathrm{Ku} / \mathrm{Nau})^{0.5}$ as Mineralocorticoid activity & 3,2 & ,032 & ,395 & 4,8 &, 000031 \\
\hline Magnesium Plasma, mM/L & 2,8 & ,056 & ,323 & 4,4 &, 000016 \\
\hline Baevsky Stress Index normalized by age, Z & 3,9 & 016 & 243 & 4,4 & ,000002 \\
\hline VLD LP Cholesterol, mM/L & 2,4 & 085 & ,202 & 4,2 & ,000002 \\
\hline Triangulary Index HRV, units & 2,0 & 133 & 172 & 4,0 & ,000002 \\
\hline Body Mass Index, kg/m² & 3,1 & 041 & 134 & 4,0 & ,000001 \\
\hline $\mathrm{AMo} / \mathrm{MxDMn}$, units & 2,6 & 066 & 107 & 4,0 &, 000000 \\
\hline Gallbladder Volume basal, $\mathrm{mL}$ & 2,7 & 066 & 086 & 4,0 &, 000000 \\
\hline Calcium Excretion, mM/24h & 2,9 &, 051 & ,066 & 4,1 &, 000000 \\
\hline Chloride Urine, $\mathrm{mM} / \mathrm{L}$ & 2,1 &, 121 & 054 & 4,0 &, 000000 \\
\hline Cortisol Plasma, nM/L & 2,1 & ,119 & 044 & 4,0 &, 000000 \\
\hline Creatinine Excretion, $\mathrm{mM} / 24 \mathrm{~h}$ & 2,7 & 065 & 034 & 4,1 &, 000000 \\
\hline (VLF+LF)/HF HRV & 1,4 &, 254 & 029 & 4,0 &, 000000 \\
\hline RMSSD HRV, msec & 1,6 & ,219 &, 025 & 3,9 &, 000000 \\
\hline Urea Excretion, $\mathrm{mM} / 24 \mathrm{~h}$ & 1,5 & 232 & 021 & 3,8 &, 000000 \\
\hline Sodium Urine, mM/L & 1,3 & ,297 & 018 & 3,7 & ,000000 \\
\hline VLF HRV, \% & 1,8 & 175 & 014 & 3,7 & ,000000 \\
\hline LF HRV, $\mathrm{msec}^{2}$ & 1,7 & 196 & 011 & 3,7 & ,000000 \\
\hline Baevsky Stress Index, ln units & 1,8 &, 185 & 009 & 3,7 &, 000000 \\
\hline Age, years & 1,4 & 269 & 007 & 3,7 & ,000001 \\
\hline SDNN HRV, msec & 2,2 & ,126 &, 003 & 4,1 &, 000000 \\
\hline Gallbladder Volume after $30 \mathrm{~min}, \mathrm{~mL}$ & 3,7 & 034 &, 002 & 4,5 &, 000000 \\
\hline $\mathrm{pNN}_{50} \mathrm{HRV}, \%$ & 1,5 & ,257 & 002 & 4,5 &, 000000 \\
\hline Testosterone normalized by sex\&age, Z & 1,6 & 241 & 001 & 4,5 & ,000000 \\
\hline Calcium Plasma, mM/L & 2,7 & 088 & 001 & 4,9 &, 000000 \\
\hline Urea Plasma, mM/L & 6,7 & ,006 & 000 & 6,2 &, 000000 \\
\hline Baevsky Activity Regulatory Systems Index & 2,3 & 139 &, 000 & 7,4 &, 000000 \\
\hline Total Power HRV, $\mathrm{msec}^{2}$ & 3,7 &, 063 &, 000 & 9,7 &, 000000 \\
\hline Phosphate Plasma, mM/L & 1,5 & ,285 & 000 & 9,9 & ,000000 \\
\hline LFnu HRV, \% & 4,4 & 071 & 000 & 13,1 & ,000000 \\
\hline
\end{tabular}

Then, the 31-dimensional space of discriminant variables is transformed into 3-dimensional space of canonical roots. The canonical correlation coefficient is for Root 10,999 (Wilks' $\Lambda=0,000001 ; X^{2}=310 ; p<10^{-6}$ ), for Root 20,992 (Wilks' $\Lambda=0,000923 ; \mathrm{X}_{(70)}^{2}=161 ; \mathrm{p}<10^{-6}$ ) and for Root 30,972 (Wilks' $\Lambda=0,0546$; 
$\left.\mathrm{X}_{(34)}^{2}=67 ; \mathrm{p}=0,0006\right)$. The major root contains $90 \%$ of discriminative properties, the second $-8 \%$ and the minor - only $2 \%$.

Table 3 presents standardized and raw coefficients for discriminant variables and constants which are necessary for the calculation of the discriminant root values for each person that enables their visualization in the information space of the roots.

Table 3 .

Standardized and raw coefficients and constants for predicting variables

\begin{tabular}{|c|c|c|c|c|c|c|}
\hline Coefficients & \multicolumn{3}{|c|}{ Standardized } & \multicolumn{3}{|c|}{ Raw } \\
\hline $\begin{array}{l}\text { Variables currently in the } \\
\text { model }\end{array}$ & Root 1 & Root 2 & Root 3 & Root 1 & Root 2 & Root 3 \\
\hline $\begin{array}{l}(\mathrm{Cap} \cdot \mathrm{Pp})^{-0,5} \text { as Calcitonin Ac- } \\
\text { tivity }\end{array}$ & $-55,92$ & $-5,07$ & 13,67 & $-832,2$ & $-75,48$ & 203,4 \\
\hline $\begin{array}{l}(\mathrm{Ku} / \mathrm{Nau})^{0.5} \text { as } \\
\text { Mineralocorticoid activity }\end{array}$ & 19,18 & ,13 & $-1,56$ & 216,3 & 1,413 & $-17,59$ \\
\hline Magnesium Plasma, mM/L & 2,84 & ,62 & $-1,28$ & 70,64 & 15,33 & $-31,92$ \\
\hline $\begin{array}{l}\text { Baevskiy's Stress Index nor- } \\
\text { malized by age, Z }\end{array}$ & 77,27 & 11,61 & $-5,30$ & 24,84 & 3,732 & $-1,705$ \\
\hline VLD LP Cholesterol, mM/L & $-32,42$ &,- 30 & 2,02 & $-149,5$ & $-1,395$ & 9,300 \\
\hline Triangulary Index HRV, units & 31,72 & $-6,41$ & $-7,54$ & 7,952 & $-1,608$ & $-1,889$ \\
\hline Body Mass Index, $\mathrm{kg} / \mathrm{m}^{2}$ & $-3,76$ & $-4,77$ & $-2,51$ & $-1,121$ & $-1,423$ &,- 750 \\
\hline AMo/MxDMn, units & $-75,05$ & $-4,51$ & $-1,49$ &,- 271 &,- 016 &,- 005 \\
\hline Gallbladder Volume basal, $\mathrm{mL}$ & 13,75 & $-1,90$ & $-1,75$ &, 903 &,- 125 &,- 115 \\
\hline Calcium Excretion, mM/24h & $-32,44$ & ,66 & 2,17 & $-13,72$ & ,278 & ,919 \\
\hline Chloride Urine, mM/L & 18,99 & $-1,82$ & $-1,26$ & 1,046 &,- 100 &,- 069 \\
\hline Cortisol Plasma, nM/L & $-18,67$ & $-1,80$ & 6,84 &,- 073 &,- 007 &, 027 \\
\hline Creatinine Excretion, $\mathrm{mM} / 24 \mathrm{~h}$ & 2,70 & $-2,19$ & 3,73 & 860 &,- 699 & 1,191 \\
\hline$(\mathrm{VLF}+\mathrm{LF}) / \mathrm{HF}$ HRV & $-10,35$ &, 11 & $-1,94$ &,- 802 & ,008 &,- 150 \\
\hline RMSSD HRV, msec & 6,08 & 7,14 & $-21,16$ & ,351 & ,412 & $-1,221$ \\
\hline Urea Excretion, mM/24h & 25,32 & 4,91 & $-7,14$ & ,115 & 022 &,- 032 \\
\hline Sodium Urine, mM/L & 6,66 &,- 74 & 1,71 & ,264 &,- 029 & ,068 \\
\hline VLF HRV, \% & 29,49 & ,34 & $-5,74$ & 1,797 & ,021 &,- 350 \\
\hline LF HRV, $\mathrm{msec}^{2}$ & 27,81 & 10,73 & 2,85 & 029 & 011 &, 003 \\
\hline Baevsky Stress Index, ln units & 13,17 & $-20,23$ & $-8,41$ & 16,66 & $-25,58$ & $-10,64$ \\
\hline Age, years & 5,05 & 8,59 &, 69 &, 388 &, 659 &, 053 \\
\hline SDNN HRV, msec & $-87,55$ & $-28,24$ & $-3,20$ & $-4,693$ & $-1,514$ &,- 171 \\
\hline $\begin{array}{l}\text { Gallbladder Volume after } 30 \\
\text { min, mL }\end{array}$ & 5,14 & 4,47 &,- 32 &, 701 & ,609 &,- 044 \\
\hline $\mathrm{pNN}_{50} \mathrm{HRV}, \%$ &, 77 & $-10,25$ & 19,24 & ,062 &,- 817 & 1,534 \\
\hline $\begin{array}{l}\text { Testosterone normalized by } \\
\text { sex\&age, Z }\end{array}$ & 3,21 & $-5,89$ & 4,38 & 1,951 & $-3,577$ & 2,659 \\
\hline Calcium Plasma, $\mathrm{mM} / \mathrm{L}$ & $-20,50$ & $-3,30$ & 3,41 & $-111,0$ & $-17,86$ & 18,46 \\
\hline Urea Plasma, mM/L & $-7,01$ & ,60 & $-2,77$ & $-5,717$ & ,489 & $-2,257$ \\
\hline $\begin{array}{l}\text { Baevsky Activity Regulatory } \\
\text { Systems Index }\end{array}$ & $-22,84$ & $-6,50$ & 4,41 & $-8,709$ & $-2,478$ & 1,681 \\
\hline Total Power HRV, $\mathrm{msec}^{2}$ & 46,72 & 15,99 & $-3,72$ & ,023 & ,008 &,- 002 \\
\hline Phosphate Plasma, mM/L & $-15,59$ & $-7,32$ & 7,30 & $-82,68$ & $-38,81$ & 38,74 \\
\hline \multirow[t]{4}{*}{ LFnu HRV, \% } & $-4,77$ & $-1,26$ & 2,35 &,- 302 &,- 079 & ,149 \\
\hline & & \multicolumn{2}{|c|}{ Constants } & 659,1 & 366,1 & 23,19 \\
\hline & & \multicolumn{2}{|c|}{ Eigenvalues } & 652,7 & 58,13 & 17,32 \\
\hline & \multicolumn{3}{|c|}{ Cumulative Properties } & ,90 & 98 & 1,00 \\
\hline
\end{tabular}


Table 4 shows the correlation coefficients of discriminant variables-predictors with canonical discriminant roots, the cluster centroids of roots as well as the values of the discriminant variables-predictors.

Table 4.

Correlations variables-canonical roots, means of roots and variables-predictors

\begin{tabular}{|c|c|c|c|c|c|c|c|c|c|}
\hline \multirow{2}{*}{\begin{tabular}{|c|}
$\begin{array}{c}\text { Variables currently } \\
\text { in the model }\end{array}$ \\
Root $1(90 \%)$ \\
\end{tabular}} & \multicolumn{3}{|c|}{$\begin{array}{c}\text { Correlations } \\
\text { Variables-Roots }\end{array}$} & \multirow{2}{*}{$\begin{array}{c}\mathrm{S} / \mathrm{S} \\
(10) \\
-42,4 \\
\end{array}$} & \multirow{2}{*}{$\begin{array}{c}\mathrm{N} / \mathrm{S} \\
(2) \\
-18,0\end{array}$} & \multirow{2}{*}{$\begin{array}{l}\mathrm{N}-/ \mathrm{N} \\
(14)\end{array}$} & \multirow{2}{*}{$\begin{array}{l}\mathrm{N} / \mathrm{N} \\
(18) \\
+19,0\end{array}$} & \multirow[t]{2}{*}{$\begin{array}{c}\text { Norm } \\
(30)\end{array}$} & \multirow[t]{2}{*}{$\mathrm{Cv}$} \\
\hline & R1 & R2 & R3 & & & & & & \\
\hline LFnu HRV, \% & ,012 &,- 032 &,- 023 & 61,0 & 67,9 & 80,2 & 72,3 & 66 & 0,210 \\
\hline VLF HRV, \% & ,008 & ,003 &,- 051 & 41,5 & 62,7 & 54,8 & 51,7 & 53,4 & 0,378 \\
\hline (VLF+LF)/HF HRV &, 008 &,- 039 &,- 057 & 6,1 & 17,5 & 22,0 & 12,0 & 8,2 & 0,506 \\
\hline $\begin{array}{l}\text { Magnesium Plasma, } \\
\mathrm{mM} / \mathrm{L}\end{array}$ & ,011 & ,045 & ,056 & 0,82 & 0,82 & 0,82 & 0,86 & 0,90 & 0,056 \\
\hline $\begin{array}{l}\text { Gallbladder Volume } \\
\text { basal, mL }\end{array}$ & ,008 & ,021 &,- 006 & 43 & 33 & 52 & 55 & 46 & 0,230 \\
\hline $\begin{array}{l}\text { Creatinine Excre- } \\
\text { tion, } \mathrm{mM} / 24 \mathrm{~h}\end{array}$ & ,007 & ,025 & ,055 & 6,85 & 5,23 & 6,52 & 8,58 & 11,0 & 0,300 \\
\hline $\begin{array}{l}\text { Testosterone nor- } \\
\text { malized, Z }\end{array}$ &,- 017 & ,007 & ,025 & $+2,80$ & $+1,49$ & $+1,07$ & $+1,09$ & $\mathbf{0}$ & \\
\hline $\begin{array}{l}\text { VLD LP Cholester- } \\
\text { ol, mM/L }\end{array}$ &,- 011 &,- 036 & ,028 & 0,77 & 0,49 & 0,70 & 0,58 & 0,53 & 0,335 \\
\hline Urea Plasma, mM/L &,- 009 &,- 021 &,- 003 & 6,61 & 6,09 & 6,34 & 5,79 & 5,00 & 0,330 \\
\hline $\begin{array}{l}\text { Calcium Excretion, } \\
\mathrm{mM} / 24 \mathrm{~h}\end{array}$ &,- 009 & ,002 &,- 023 & 4,90 & 5,50 & 4,03 & 3,71 & 4,38 & 0,214 \\
\hline Root $2(8 \%)$ & R1 & R2 & R3 & $+0,7$ & $+13,9$ & $-9,8$ & $+5,7$ & & \\
\hline $\begin{array}{l}(\mathrm{Cap} \cdot \mathrm{Pp})^{-0,5} \text { as } \\
\text { Calcitonin }\end{array}$ &,- 005 & ,073 & ,074 & 0,67 & 0,70 & 0,61 & 0,70 & 0,60 & 0,167 \\
\hline $\begin{array}{l}\text { Urea Excretion, } \\
\mathrm{mM} / 24 \mathrm{~h}\end{array}$ &,- 012 &, 020 &,- 047 & 614 & 799 & 481 & 483 & 458 & 0,186 \\
\hline SDNN HRV, msec & ,001 & ,037 & ,037 & 45,5 & 45,5 & 38,4 & 53,5 & 55 & 0,201 \\
\hline $\begin{array}{l}\text { Triangulary Index } \\
\text { HRV, units }\end{array}$ & 001 &, 019 & ,036 & 11,1 & 10,0 & 9,8 & 11,7 & 11,2 & 0,217 \\
\hline $\begin{array}{l}\text { Phosphate Plasma, } \\
\mathrm{mM} / \mathrm{L}\end{array}$ & ,003 &,- 060 &,- 040 & 0,99 & 0,99 & 1,17 & 0,97 & 1,20 & 0,167 \\
\hline $\begin{array}{l}\text { Body Mass Index, } \\
\mathrm{kg} / \mathrm{m}^{2}\end{array}$ &,- 002 &,- 054 &,- 040 & 26,0 & 25,5 & 28,9 & 25,6 & 24,2 & \\
\hline $\begin{array}{l}\text { Calcium Plasma, } \\
\mathrm{mM} / \mathrm{L}\end{array}$ & ,006 &,- 053 &,- 079 & 2,11 & 2,28 & 2,33 & 2,13 & 2,30 & 0,065 \\
\hline $\begin{array}{l}\text { Baevsky Stress In- } \\
\text { dex, Z }\end{array}$ & ,002 &,- 041 &,- 019 & $+0,54$ & $-0,18$ & $+3,24$ & $+0,10$ & $\mathbf{0}$ & \\
\hline $\begin{array}{l}\text { Baevsky Activity } \\
\text { Reg Syst Ind }\end{array}$ &,- 000 &,- 035 & ,016 & 3,50 & 1,50 & 4,31 & 2,94 & 1,50 & 0,624 \\
\hline $\begin{array}{l}\text { Baevsky Stress In- } \\
\text { dex, ln units }\end{array}$ &,- 001 &,- 039 &,- 028 & 4,82 & 4,82 & 5,15 & 4,63 & 4,91 & \\
\hline $\begin{array}{l}(\mathrm{Ku} / \mathrm{Nau})^{0.5} \text { as Min- } \\
\text { eralocorticoid }\end{array}$ & ,008 &,- 036 & ,065 & 0,54 & 0,41 & 0,59 & 0,57 & 0,54 & 0,269 \\
\hline $\begin{array}{l}\text { Gallbladder Vol af- } \\
\text { ter } 30 \mathrm{~min}, \%\end{array}$ &,- 004 &,- 033 &,- 012 & 65,8 & 60,9 & 68,1 & 67,5 & 62,0 & 0,081 \\
\hline AMo/MxDMn, units &,- 000 &,- 030 &,- 007 & 279 & 209 & 382 & 238 & 142 & 0,307 \\
\hline Age, years &,- 003 &,- 018 &, 010 & 51,4 & 45,0 & 51,4 & 48,0 & 49,7 & 0,256 \\
\hline Root $3(2 \%)$ & R1 & R2 & R3 & $+2,4$ & $-16,3$ & $-1,8$ & $+1,9$ & & \\
\hline $\begin{array}{l}\text { Chloride Urine, } \\
\mathrm{mM} / \mathrm{L}\end{array}$ & ,016 &,- 013 & ,085 & 97,5 & 73 & 106 & 114 & 120 & 0,172 \\
\hline
\end{tabular}




\begin{tabular}{|l|c|c|c|c|c|c|c|c|c|}
\hline $\begin{array}{l}\text { Variables currently } \\
\text { in the model }\end{array}$ & \multicolumn{2}{|c|}{$\begin{array}{c}\text { Correlations } \\
\text { Variables-Roots }\end{array}$} & $\begin{array}{c}\text { S/S } \\
(10)\end{array}$ & $\begin{array}{c}\text { N/S } \\
(2)\end{array}$ & $\begin{array}{c}\text { N-/N } \\
(14)\end{array}$ & $\begin{array}{c}\text { N/N } \\
(18)\end{array}$ & $\begin{array}{c}\text { Norm } \\
(30)\end{array}$ & Cv \\
\hline $\begin{array}{l}\text { Sodium Urine, } \\
\text { mM/L }\end{array}$ &, 003 &,- 012 & $\mathbf{0 4 9}$ & 121 & $\mathbf{9 5}$ & 122 & 123 & 161 & 0,211 \\
\hline RMSSD HRV, msec &,- 004 &, 020 & $\mathbf{, 0 4 6}$ & 34,6 & $\mathbf{2 1 , 5}$ & 22,4 & 30,6 & $\mathbf{2 8}$ & 0,392 \\
\hline pNN $_{50}$ HRV, \% &,- 003 &, 016 & $\mathbf{, 0 4 4}$ & 13,6 & $\mathbf{4 , 0}$ & 6,0 & 11,0 & $\mathbf{9 , 2}$ & 1,518 \\
\hline $\begin{array}{l}\text { Total Power HRV, } \\
\text { msec }^{2}\end{array}$ &, 003 &, 033 & $\mathbf{, 0 4 3}$ & 2644 & $\mathbf{1 6 9 5}$ & 3310 & 2029 & $\mathbf{2 4 7 7}$ & 0,399 \\
\hline LF HRV, msec $^{2}$ &, 003 &, 019 & $\mathbf{, 0 4 0}$ & 929 & $\mathbf{5 1 8}$ & 685 & 1169 & $\mathbf{6 2 7}$ & 0,529 \\
\hline $\begin{array}{l}\text { Cortisol Plasma, } \\
\text { nM/L }\end{array}$ &, 001 &,- 032 & $-\mathbf{0 6 6 7}$ & 542 & 762 & 718 & 528 & $\mathbf{4 0 5}$ & 0,315 \\
\hline
\end{tabular}

In general, all four clusters on the planes of the discriminant roots are very clearly delineated, which is documented by calculating the Mahalanobis distances (Table 4)

Table 4.

Squared Mahalanobis distances between clusters, F-values $(\mathrm{df}=36,5)$ and $p$-levels

\begin{tabular}{|c|c|c|c|c|}
\hline Clusters & N/N & S/S & N/S & N-/N \\
\hline N/N & 0 & 4172 & 1941 & 402 \\
\hline S/S & $\mathbf{8 5 , 3}$ & 0 & 1229 & 2984 \\
& $10^{-4}$ & & & \\
\hline N/S & $\mathbf{6 , 4}$ & 3,8 & 0 & 1619 \\
& 0,024 & 0,068 & & \\
\hline N-/N & $\mathbf{1 0 , 3}$ & $\mathbf{5 5 , 1}$ & $\mathbf{5 , 2}$ & 0 \\
& 0,008 & $10^{-4}$ & 0,036 & \\
\hline
\end{tabular}

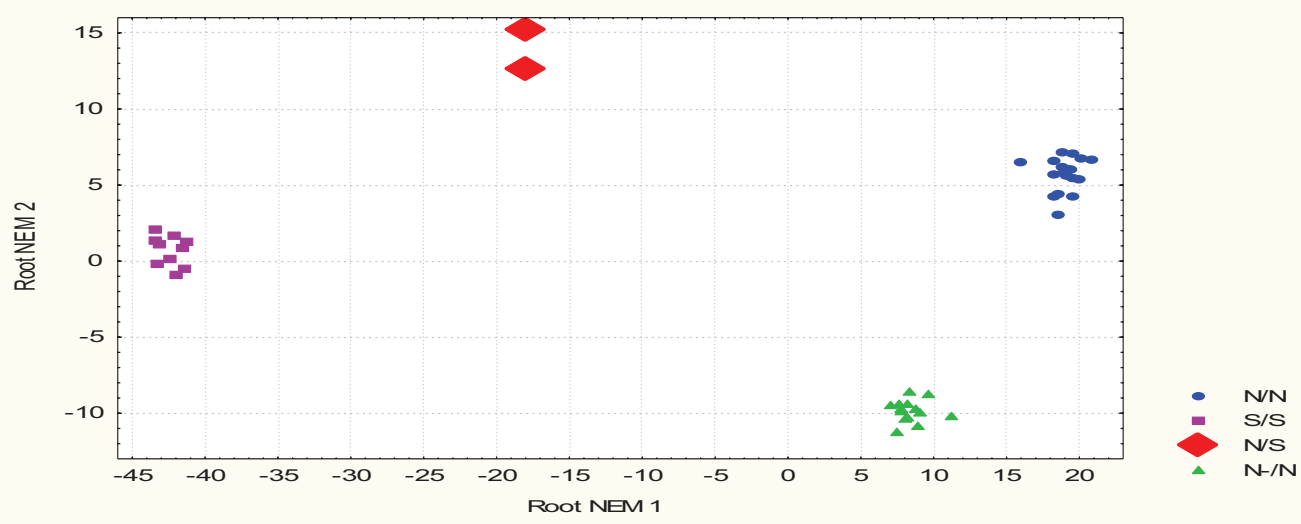

Fig. 2. Scatterplot of individual values of the first and second roots in which condensed information about initial values of vegetative, endocrine and metabolic parameters as predictors for the members of the four clusters

The predictors of the S/S type immune response are the minimum for sample values of parameters that are positively related to the first root and the maximum values for inversely correlating parameters. Conversely, the N/N type immune response is preceded by the maximum/minimum values of other predictors (Fig. 2).

The $\mathrm{N}-/ \mathrm{N}$ type immune response occurs in individuals with a minimum of initial values parameters that are directly related to the second root and maximum values of the parameters that are inversely correlated with the root. In members of the N/S immune response cluster, localized in the opposite zone of the axis of the second root, these parameters are usually maximum/minimum. 
Additional predictors of this type of immune response are the minimum values of parameters that correlate positively with the third root as well as the maximum level of plasma cortisol.

The ultimate goal of the discriminant analysis is realized with the help of classifying functions (Table 5).

Table 5 .

Coefficients and constants for the classification of the functions of clusters

\begin{tabular}{|c|c|c|c|c|}
\hline Clusters & $\mathrm{N} / \mathrm{N}$ & $\mathrm{S} / \mathrm{S}$ & $\mathrm{N} / \mathrm{S}$ & $\mathrm{N}-/ \mathrm{N}$ \\
\hline Variables & $\mathrm{p}=, 409$ & $\mathrm{p}=, 227$ & $\mathrm{p}=, 045$ & $\mathrm{p}=, 318$ \\
\hline$(\mathrm{Cap} \cdot \mathrm{Pp})^{-0,5}$ as Calcitonin Activity & 571174 & 622740 & 597658 & 580376 \\
\hline$(\mathrm{Ku} / \mathrm{Nau})^{0.5}$ as Mineralocorticoid activity & -138139 & -151431 & -145811 & -140377 \\
\hline Magnesium Plasma, mM/L & -43049 & -47478 & -44958 & -43915 \\
\hline Baevsky Stress Index normalized by age, $\mathrm{Z}$ & -17669 & -19214 & -18527 & -17983 \\
\hline VLD LP Cholesterol, mM/L & 96783 & 105972 & 102135 & 98347 \\
\hline Triangulary Index HRV, units & -4453 & -4934 & -4726 & -4505 \\
\hline Body Mass Index, kg/m² & 1283 & 1359 & 1327 & 1320 \\
\hline AMo/MxDMn, units & 184,6 & 201,4 & 194,6 & 187,8 \\
\hline Gallbladder Volume basal, $\mathrm{mL}$ & $-522,4$ & $-577,2$ & $-554,7$ & $-529,5$ \\
\hline Calcium Excretion, mM/24h & 8707 & 9548 & 9200 & 8844 \\
\hline Chloride Urine, $\mathrm{mM} / \mathrm{L}$ & $-640,5$ & $-704,2$ & $-678,7$ & $-649,7$ \\
\hline Cortisol Plasma, nM/L & 48,8 & 53,3 & 50,9 & 49,5 \\
\hline Creatinine Excretion, $\mathrm{mM} / 24 \mathrm{~h}$ & $-284,8$ & $-333,4$ & $-343,9$ & $-287,3$ \\
\hline$(\mathrm{VLF}+\mathrm{LF}) / \mathrm{HF}$ HRV & 521,4 & 570,5 & 553,8 & 530,2 \\
\hline RMSSD HRV, msec & $-313,7$ & $-337,9$ & $-301,2$ & $-319,3$ \\
\hline Urea Excretion, mM/24h & $-82,8$ & $-90,0$ & $-86,3$ & $-84,3$ \\
\hline Sodium Urine, mM/L & $-171,8$ & $-187,8$ & $-183,0$ & $-174,4$ \\
\hline VLF HRV, \% & -1128 & -1239 & -1188 & -1146 \\
\hline LF HRV, $\mathrm{msec}^{2}$ & $-23,1$ & $-24,9$ & $-24,1$ & $-23,5$ \\
\hline Baevsky Stress Index, ln units & $-585,7$ & -1485 & -1219 & $-325,5$ \\
\hline Age, years & $-528,8$ & $-555,9$ & $-538,7$ & $-543,3$ \\
\hline SDNN HRV, msec & 3659 & 3955 & 3824 & 3733 \\
\hline Gallbladder Volume after $30 \mathrm{~min}, \mathrm{~mL}$ & $-676,1$ & $-722,2$ & $-696,2$ & $-692,7$ \\
\hline $\mathrm{pNN}_{50} \mathrm{HRV}, \%$ & 203,6 & 204,7 & 166,8 & 210,1 \\
\hline Testosterone normalized by sex\&age, Z & 65,2 & $-35,2$ & $-84,5$ & 90,4 \\
\hline Calcium Plasma, mM/L & 79359 & 86271 & 82984 & 80738 \\
\hline Urea Plasma, mM/L & 3543 & 3890 & 3799 & 3603 \\
\hline Baevsky Activity Regulatory Systems Index & 6560 & 7107 & 6831 & 6684 \\
\hline Total Power HRV, $\mathrm{msec}^{2}$ & $-17,9$ & $-19,3$ & $-18,7$ & $-18,3$ \\
\hline Phosphate Plasma, mM/L & 70974 & 76264 & 73013 & 72307 \\
\hline LFnu HRV, \% & 222,0 & 241,0 & 229,8 & 225,9 \\
\hline Constant & -289637 & -332624 & -311642 & -302238 \\
\hline
\end{tabular}

As we can see (Table 6), all types of immune response to adaptogenic balneotherapy are predicted unmistakably.

Classification of Matrix

Rows: Observed classifications; Columns: Predicted classifications

\begin{tabular}{|c|c|c|c|c|c|}
\hline & Percent & $\mathrm{N} / \mathrm{N}$ & $\mathrm{S} / \mathrm{S}$ & $\mathrm{N} / \mathrm{S}$ & $\mathrm{N}-/ \mathbf{N}$ \\
\cline { 3 - 6 } & correct & $\mathrm{p}=, 409$ & $\mathrm{p}=, 227$ & $\mathrm{p}=, 045$ & $\mathrm{p}=, 318$ \\
\hline N/N & 100 & 18 & 0 & 0 & 0 \\
\hline S/S & 100 & 0 & 10 & 0 & 0 \\
\hline
\end{tabular}




\begin{tabular}{|c|c|c|c|c|c|}
\hline & Percent & $\mathrm{N} / \mathrm{N}$ & $\mathrm{S} / \mathrm{S}$ & $\mathrm{N} / \mathrm{S}$ & $\mathrm{N}-/ \mathbf{N}$ \\
\cline { 3 - 6 } & correct & $\mathrm{p}=, 409$ & $\mathrm{p}=, 227$ & $\mathrm{p}=, 045$ & $\mathrm{p}=, 318$ \\
\hline N/S & 100 & 0 & 0 & 2 & 0 \\
\hline $\mathbf{N}-/ \mathbf{N}$ & 100 & 0 & 0 & 0 & 14 \\
\hline Total & 100 & 18 & 10 & 2 & 14 \\
\hline
\end{tabular}

Conclusion. The previously revealed variety of immune responses to adaptogenic balneotherapy is quite strictly conditioned by the initial state of the neuroendocrine-immune complex, microbiota, metabolism as well as the sex and age of patients.

\section{Acknowledgement}

We'd like to express sincere gratitude to the administration of JSC "Truskavets' kurort" and "Truskavets' SPA" as well as the clinical sanatorium "Moldova" for help in conducting this investigation.

\section{Accordance to ethics standards}

Tests on patients are conducted in accordance with positions of Helsinki Declaration 1975, revised and complemented in 2002 and the directive of National Committee on ethics of scientific researches. During the realization of tests from all participants the informed consent has been received and all measures for providing the anonymity of participants have been used. There is not any conflict of interests among the authors.

\section{REFERENCES}

1. Struk ZD, Mel'nyk OI, Mysakovets' OG. Individual immune responses to adaptogens and their predictors. In: Rehabilitation Medicine and Health-Resort Institutions Development. Proceedings of the 19th International Applied Research Conference (Kyiv, 11-12 December 2019). Edited by O. Gozhenko, W. Zukow. Kyiv: Toruń. 2019: 83-4.

2. Struk ZD, Mel'nyk OI, Zukow W, Popovych IL. The diversity of immune reactions to balneotherapy and their accompaniments. Journal of Education, Health and Sport. 2019; 9(11): 349-73.

3. Mel'nyk OI, Struk ZD. Predictors of individual immune responses to adaptogens. Experimental and Clinical Physiology and Biochemistry. 2019; 88(4): 5-15.

4. Mel'nyk OI, Struk ZD, Zukow W, Popovych IL. Vegetative, endocrine and metabolic accompaniments of individual immune responses to adaptogenic balneotherapy. Journal of Education, Health and Sport. 2019; 9(12): 207-29.

5. Sternberg EM. Neural regulation of innate immunity: a coordinated nonspecific response to pathogens. Nature Reviews Immunology. 2006; 6(4): 318-8.

6. Uchakin PN, Uchakina ON, Tobin BV, Yershov FI. Neuroendocrine Immunomodulation. Vestnik Ross AMN. 2007; 9: 26-32. [in Russian]

7. Thayer JF, Sternberg EM. Neural aspects of immunomodulation: Focus on the vagus nerve. Brain Behav Immun. 2010; 24(8): 1223-8.

8. Pavlov VA, Chavan SS, Tracey KJ. Molecular and functional neuroscience in immunity. Annual Review of Immunology. 2018; 36: 783-812.

9. Gozhenko AI, Zukow W, Polovynko IS, Zajats LM, Yanchij RI, Portnichenko VI et al. Individual Immune Responses to Chronic Stress and their Neuro-Endocrine Accompaniment. RSW. UMK. Radom. Torun. 2019: 200 p.

10. Gozhenko AI. Functional-metabolic continuum. Journal of NAMS of Ukraine. 2016; 22(1): 3-8. [in Russian]

11. Barylyak LG, Tsymbryla VV, Zukow W, Popovych IL. Relationships between parameters of plasma lipoproteines profile and heart rate variability. Journal of Education, Health and Sport. 2019; 9(12): 238-53.

12. Struk ZD, Mel'nyk OI, Zukow W, Popovych IL. Vegetative and endocrine predictors of individual immune responses to adaptogenic balneotherapy. Journal of Education, Health and Sport. 2020; 10(1): 218-25.

13. Struk ZD, Mel'nyk OI, Zukow W, Popovych IL. Metabolic predictors of individual immune responses to adaptogenic balneotherapy. Journal of Education, Health and Sport. 2020; 10(2): $223-30$.

14. Baevskiy RM, Ivanov GG. Heart Rate Variability: theoretical aspects and possibilities of clinical application. Ultrazvukovaya i funktsionalnaya diagnostika. 2001; 3: 106-27. [in Russian]

15. Heart Rate Variability. Standards of Measurement, Physiological Interpretation, and Clinical Use. Task Force of ESC and NASPE. Circulation. 1996; 93(5): 1043-65.

16. Berntson GG, Bigger JT jr, Eckberg DL, Grossman P, Kaufman PG, Malik Met al. Heart Rate Variability: Origines, methods and interpretive caveats. Psychophysiology. 1997; 34: 623-48. 
17. Popadynets' OO, Gozhenko AI, Zukow W, Popovych IL. Relationships between the entropies of EEG, HRV, immunocytogram and leukocytogram. Journal of Education, Health and Sport. 2019; 9(5): 651-66.

18. Hiller $G$. Test for the quantitative determination of HDL cholesterol in EDTA plasma with Reflotron. Clinical Chemistry. 1987; 33: 895-8.

19. Goryachkovskiy AM. Clinical Biochemistry. Odessa: Astroprint; 1998: 608 p. [in Russian]

20. Klecka WR. Discriminant Analysis [translated from English to Russian] (Seventh Printing, 1986). In: Factor, Discriminant and Cluster Analysis. Moskva: Finansy i Statistika; 1989: 78-138.

Стаття надійшла до редколегії 18.02.2020

\title{
RESEARCH ARTICLE \\ The variety of immune responses to adoptogenetic balneotherapy is strictly deterministic
}

\author{
O.I. MEL'NYK', Z.D. STRUK ${ }^{2}$ \\ ${ }^{1}$ Danylo Halyts'kyĭ National Medical University, L'viv, Ukraine \\ E-mail: omelnyk7@gmail.com

\section{${ }^{2}$ Ukrainian Scientific Research Institute of Medicine for Transport, Odesa, Ukraine}

Four variants of the immune responses to adaptogenic balneotherapy have been identified. It is shown that the immunotropic effect of balneotherapy on certain individuals is not effective enough and in some cases even unfavorable. All four variants of immune responses are virtually unmistakably predicted by a set of 20 immune and microbiota predictors. Each type of immune response is characterized by a specific vegetative, endocrine and metabolic support. In the next study the discriminant analysis revealed that the constellation $8 \mathrm{HRV}$ and 5 endocrine parameters as well as gender of the patient predicts the nature of the immune response with an accuracy of $90,9 \%$. As soon as we found out 16 metabolic parameters as the predictors which, taken together, determine the nature of the immune response with an accuracy of $95,5 \%$. Standing on the positions of determinism, we assumed that taking into account all the recorded initial neuro-endocrine and metabolic parameters of the body would allow to predict the immune response of each patient unmistakably. Testing this hypothesis was the purpose of this study.

The object of observation was 34 men and 10 women aged 24-70, who came to the Truskavets' spa for the treatment of chronic pyelonephritis combined with cholecystitis in remission. The state of the autonomic nervous system is estimated by parameters of HRV ("CardioLab+HRV"). To assess the endocrine status we determined plasma levels of principal adaptation hormones: Cortisol, Testosterone and Triiodothyronine (ELISA with the use of analyzer "RT-2100C"). The metabolic panel amounted to: plasma lipoproteines; the plasma and daily urine levels of the electrolytes (calcium, magnesium, phosphates, chloride, sodium and potassium; nitrous metabolites (creatinine, urea, uric acid). According to the parameters of electrolyte exchange, hormonal activity was evaluated: parathyroid, calcitonin and mineralocorticoid.

Following the accepted algorithm, the forward stepwise method revealed 31 parameters as the predictors, among them: $13 \mathrm{HRVs}, 4$ endocrine, 11 metabolic as well as 2 parameters of cholekinetics and age. All types of immune response to adaptogenic balneotherapy are predicted unmistakably.

The previously revealed variety of immune responses to adaptogenic balneotherapy is quite strictly conditioned by the initial state of the neuroendocrine-immune complex, microbiota, metabolism as well as the sex and age of patients.

Key words: chronic inflammation, adaptogenic balneotherapy, immune responses, HRV, hormones, metabolism. 